Koen S. Simons

Peter Pickkers

Chantal P. Bleeker-Rovers

Wim J. G. Oyen

Johannes G. van der Hoeven

\section{F-18-fluorodeoxyglucose positron emission tomography combined with CT in critically ill patients with suspected infection}

Received: 22 April 2009

Accepted: 2 October 2009

Published online: 22 October 2009

(C) The Author(s) 2009. This article is published with open access at Springerlink.com
K. S. Simons · P. Pickkers (®) ·

J. G. van der Hoeven

Department of Intensive Care

Medicine (632), Radboud University

Nijmegen Medical Centre, Nijmegen,

The Netherlands

e-mail: p.pickkers@ic.umcn.nl

Tel.: +31-24-3617273

Fax: +31-24-2541612

\section{P. Bleeker-Rovers}

Division of Infectious Diseases, Department of General Internal Medicine, Radboud University Nijmegen Medical Centre, Nijmegen, The Netherlands

W. J. G. Oyen

Department of Nuclear Medicine,

Radboud University Nijmegen Medical

Centre, Nijmegen, The Netherlands
P. Pickkers - C. P. Bleeker-Rovers · W. J. G. Oyen - J. G. van der Hoeven Nijmegen Institute for Infection, Inflammation and Immunity (N4i), Nijmegen, The Netherlands

\begin{abstract}
Purpose: To assess the value of F-18-fluorodeoxyglucose positron emission tomography (FDGPET) combined with CT in critically ill patients suspected of having an infection. Methods: FDG-PET CT scans requested for evaluation of a suspected infection or inflammatory process in critically ill, mechanically ventilated patients were analyzed (blinded for the final clinical diagnosis) and compared with clinical follow-up. Results: Thirty-five FDG-PET/CT scans performed in 33 ICU patients (28 adults and 5 children), median age 58 years (range 1 month-72 years), were analyzed. Twenty-one FDG-PET/CT scans were true positive. Three FDG-PET/CT scans were considered false positive, in one case leading to additional diagnostic procedures
\end{abstract}

(specificity 79\%). Additionally, 11 true negatives were found (sensitivity $100 \%$ ), leading to an overall accuracy of $91 \%$. Conclusions: FDG-PET/ CT scanning is of additional value in the evaluation of suspected infection in critically ill patients in whom conventional diagnostics did not lead to a diagnosis. Apart from the high accuracy, in this study it appeared that, in addition to conventional diagnostic techniques that were routinely performed, a normal FDG-PET/CT ruled out important infections requiring prolonged antibiotic therapy or drainage. Since sensitivity is lower in highly metabolic active tissues (e.g., endocarditis, meningitis), the FDG-PET/CT scan is not suited to detect infections in these tissues.

Keywords Positron emission tomography · Critical illness . Intensive care · Infection · Diagnosis

\section{Introduction}

At some point in time during their stay in the ICU, many critically ill patients are suspected of having an infectious process. While in most cases, a diagnosis can be reached on clinical, radiological or microbiological grounds, a significant number of cases remain in which a definitive diagnosis cannot be made even following extensive diagnostic testing. Timely identification and localization of infectious foci is critical for appropriate treatment of these patients. However, given the limitations and side effects of conventional diagnostic procedures (e.g., excessive radiation exposure, contrast nephrotoxicity), new techniques to detect these infectious foci are still necessary.

In recent years, F-18-fluorodeoxyglucose (FDG) positron emission tomography (PET) scanning has gained 
interest. FDG-PET depicts cells with an enhanced glucose metabolism. Apart from its role in the evaluation of malignancies, the role of FDG-PET in the diagnosis of focal inflammation and infection has been studied in patients suffering from osteomyelitis [1-3], vasculitis [48], inflammatory bowel disease [9-11] and sarcoidosis $[12,13]$. Moreover, recent studies have shown its usefulness as a diagnostic tool in patients with fever of unknown origin (FUO) where more than one third of the PET scans were considered helpful in reaching a final diagnosis [1417]. In a retrospective study evaluating FDG-PET scanning in patients with bacteremia suspected of metastatic infectious complications, FDG-PET revealed clinically relevant new infectious foci in $45 \%$ of patients with a negative predictive value of $100 \%$ [18]. Importantly, in all of these studies, patients admitted to an ICU were excluded. To our knowledge, up to now the role of FDG-PET in diagnosing focal infections in the critically ill ICU patient has not been evaluated systematically.

To assess the role of FDG-PET for the evaluation of ventilated critically ill patients with suspected infection or inflammation, we evaluated the results of FDG-PET in this specific group of patients. By using an integrated PET/CT scanner, the functional (PET) and anatomical (CT) images were recorded in one session, providing direct correlation between areas of increased FDG uptake and anatomical changes in a single diagnostic procedure.

\section{Methods}

\section{Patients}

Between October 2005 and March 2008, all FDG-PET/CT scans ordered for evaluation of a suspected infection in mechanically ventilated ICU patients in a 34-bed medical and surgical ICU were analyzed. Following Dutch law, approval by an ethics committee was exempt since PET/ CT scans are frequently made in our hospital in the workup of patients with FUO, and the described results should be considered as a retrospective observational study. Only FDG-PET/CT scans were included of patients in whom a clear clinical suspicion of infection existed, indicated by at least one of the following signs: fever, leucocytosis $\left(>12 \times 10^{9} / 1\right)$, tachycardia $(>90 \mathrm{bpm})$, elevated levels of $C$-reactive protein $(>100 \mathrm{mg} / \mathrm{l})$, positive blood cultures and/or persistent vasopressor dependency. A fixed diagnostic protocol prior to the PET/CT scan was not obligatory; however, all patients underwent an extensive physical examination, laboratory investigation, culturing and chest X-ray. In most of the patients, additional diagnostic procedures (CT scanning, abdominal ultrasound, echocardiography) were performed prior to the FDG-PET/CT scan. For pragmatic reasons no selection was made based on type of suspected infection, so all ICU patients with the above-mentioned criteria were included.

\section{FDG-PET/CT}

An integrated PET/CT scanner (Siemens Biograph, Knoxville, TN) was used for data acquisition. Prior to FDG injection, patients were fasted, and glucose or insulin-containing intravenous infusions were discontinued for at least $6 \mathrm{~h}$. In all patients, glucose levels were checked and were below $11 \mathrm{mmol} / \mathrm{l}$. One hour after intravenous injection of 200-220 MBq FDG (Covidien, Petten, The Netherlands), a low-dose CT of the area between the proximal femora and the base of the skull was made for anatomical correlation and attenuation correction of the PET data.

Subsequently, emission images of the same area were acquired, using an emission time of 4 min per bed position. The FDG dose was based on the patient's weight according to the NEDPAS guidelines [19]. Immediately after performing the FDG-PET/CT scan, the results were interpreted by two staff members of the Department of Nuclear Medicine without knowledge of the results of other diagnostic tests or the final diagnosis. Disagreements were resolved by consensus.

\section{Interpretation of FDG-PET/CT scan results}

The final diagnosis, based on biopsy, positive serology, positive cultures or autopsy results, was used for comparison with FDG-PET/CT results. This diagnosis was made by the ICU staff responsible for the treatment of the patient with knowledge of the results of the FDG-PET/CT scan. The diagnosis was never based on the results of the FDG-PET/CT scan alone.

Abnormal FDG-PET/CT scans were considered helpful in establishing the diagnosis or 'true positive' when abnormal FDG-uptake in an organ or tissue corresponded with the site of infection/inflammation as determined by additional diagnostic techniques. Abnormal results were regarded as non-contributory to diagnosis or 'false positive' when the detected abnormality was considered to be unrelated to the illness causing the inflammatory response or when no final diagnosis could be made. A normal FDG-PET/CT scan was considered 'true negative' when no cause of the symptoms was identified despite an extensive diagnostic workup and clinical follow-up until ICU discharge or death. As FDG-PET is unsuitable for detection of infectious foci within the brain, the heart and kidney due to high physiological FDG uptake, FDG-PET was not evaluated for infectious foci in these organs. When patients were discharged and readmitted to the ICU, the clinical course until the second discharge was recorded. Infections diagnosed more than 2 weeks after FDG-PET/CT were not considered to be present at the time of FDG-PET/CT. A normal FDG-PET scan was considered 'false negative' when a focal infection, a focal inflammatory process or neoplasm that reasonably can be 
detected with PET/CT scanning was diagnosed by means of tissue cultures or histology that was thought to be already present at the time of the FDG-PET/CT scan.

\section{Statistical analysis}

We calculated sensitivity, specificity, positive and negative predictive values, positive and negative likelihood ratios and the overall accuracy defined as true positives + true negatives/true positives + true negatives + false positives + false negatives.

\section{Results}

From October 2005 to March 2008, 38 FDG-PET/CT scans were performed in 36 ICU patients. There were 4,180 ICU admissions during this period. No adverse effects were observed after injection of the tracer. No complications occurred during transport to and from the PET/CT scanner. One patient did not fulfill the criteria and was therefore excluded. Two patients were excluded because of a very short follow-up time of 1 day during which no definitive diagnosis was made; 1 patient died and 1 patient was transferred to the ward, so the results of 35 FDG-PET/CT scans performed in 33 patients were analyzed ( 28 adults and 5 children, 24 male and 9 female) with a median age of 58 years (range 1 month-72 years). In two patients, a second FDG-PET/CT scan was ordered. These were considered to be separate cases since the time interval between these PET/CT scans was at least 1 month.

ICU admission diagnoses of patients are illustrated in Table 1. Reasons for FDG-PET/CT scanning were persistent fever $(n=17)$, suspected septic emboli $(n=10)$, suspected mediastinitis $(n=3)$, persistent systemic inflammatory response syndrome (SIRS) $(n=3)$ and suspicion of infected intra-abdominal fluid collections $(n=2)$. In 27 of 35 cases antibiotic $(n=24)$ and/or antifungal $(n=3)$ treatment was given, and in 30 cases, fever was present at the time of the FDG-PET/CT scan with a median duration of 12 days (range 2-41 days).

Table 2 shows the prior diagnostic workup, the definite clinical diagnosis and the correlation with the PET/CT scan results of all included patients. Of 35 FDGPET/CT scans, 24 were considered abnormal, and 11 were considered normal. Of the 24 abnormal FDG-PET/ CT scans, 21 were considered true positive (Table 3). In 14 of these 21 true positives, additional investigations were performed to evaluate the PET/CT abnormalities (radiologic imaging $n=7$, consulting a specialist $n=4$, additional culturing $n=3$ ). In five cases, the results of the PET/CT scan had direct therapeutic consequences
Table 1 ICU admission diagnoses of all 33 patients

Reason for ICU admittance

Elective cardiac surgery

Complications of prior cardiac surgery

Respiratory insufficiency

Endocarditis

Septic shock

Laparotomy for

Suspected perforation

Repair of ruptured aneurysm

Evacuation of infected hematoma

Trauma

Subarachnoid hemorrhage

Epileptic seizure

Hepatic failure

Cardiogenic shock

Hypovolemic shock

(surgery $n=3$; removal of i.v. cannula $n=1$; start of antibiotic therapy $n=1$ ). Figures 1 and 2 illustrate two cases in which the results of the FDG-PET/CT scans showed unexpected abnormalities where subsequent diagnostic tests indeed showed an infection at the site of those abnormalities. True-positive FDG-PET results were ultimately confirmed by culture $(n=12)$, biopsy $(n=2)$ or autopsy $(n=1)$, and a probable confirmation was reached in six cases through radiology $(n=5)$ or clinical improvement after treatment based on the FDG-PET/CT scan results $(n=1$; Table 4$)$ described in the electronic supplement.

In 11 patients in whom additional investigations and the clinical course showed no evidence of an infection, a normal FDG-PET/CT scan was classified as 'true negative.' In one case, a patient, transferred from another ICU because of difficult weaning after a pneumococcal pneumonia, a FDG-PET/CT scan was made because of persisting fever, showing no clear abnormalities. Since the presence of an autoimmune systemic disease was suspected, additional diagnostic tests were performed, including a kidney biopsy, and a final diagnosis of systemic lupus erythematodes was made. Although the auto-immune inflammatory disease was not detected by FDG-PET/CT scan, this patient had no active infection, so he was evaluated as being true negative for infection.

In those patients in whom the FDG-PET/CT scan results were negative, median duration of clinical followup was 33 days (range 4-74 days). After ICU discharge, hospital correspondence was reviewed with a median duration of 5 months (range 1-12) after discharge off the ICU.

In three patients, positive results of the FDG-PET/CT scan were followed by several diagnostic tests, which finally showed no abnormalities and were consequently termed 'false positive.' In one patient, admitted with an infective endocarditis, a FDG-PET/CT scan, performed to 
Table 2 Diagnostic workup prior to PET/CT scan, final diagnosis and correlation with PET/CT findings in all patients; in two patients (nos. 3 and 27) two $\mathrm{PET} / \mathrm{CT}$ scans were made

\begin{tabular}{|c|c|c|c|c|c|c|c|c|c|c|c|c|c|}
\hline \multirow[t]{2}{*}{ Patient } & \multirow{2}{*}{$\begin{array}{l}\text { Sex/ } \\
\text { age }\end{array}$} & \multirow{2}{*}{$\begin{array}{l}\text { Indication } \\
\text { fo PET/ } \\
\text { CT scan }\end{array}$} & \multirow{2}{*}{$\begin{array}{l}\text { Days on } \\
\text { ICU }\end{array}$} & \multirow{2}{*}{$\begin{array}{l}\text { Day of } \\
\text { fever }\end{array}$} & \multicolumn{3}{|c|}{ Cultures } & \multirow{2}{*}{$\begin{array}{l}\text { Cardiac } \\
\text { US }\end{array}$} & \multirow{2}{*}{$\begin{array}{l}\text { Vascular } \\
\text { US }\end{array}$} & \multirow[t]{2}{*}{ CT scan } & \multirow{2}{*}{$\begin{array}{l}\text { Final } \\
\text { diagnosis }\end{array}$} & \multirow{2}{*}{$\begin{array}{l}\text { Mode of } \\
\text { diagnosis }\end{array}$} & \multirow{2}{*}{$\begin{array}{l}\text { PETCT } \\
\text { correlation }\end{array}$} \\
\hline & & & & & $\mathrm{S}$ & B & $\mathrm{U}$ & & & & & & \\
\hline 1 & $\mathrm{M} / 63$ & FUO & 24 & 24 & + & - & - & - & - & - & No infection & - & FP \\
\hline 2 & $\mathrm{M} / 50$ & Mediastinitis & 12 & 11 & + & - & - & - & - & _- & Mediastinitis & Biopsy & $\mathrm{TP}$ \\
\hline $3 a$ & $\mathrm{M} / 58$ & FUO & 10 & 10 & + & + & - & - & - & Abdomen & Phlebitis & Culture & $\mathrm{TP}$ \\
\hline $3 b$ & $\mathrm{M} / 58$ & FUO & 41 & 41 & + & + & - & - & - & - & Pneumonia & Culture & $\mathrm{TP}$ \\
\hline 4 & $\mathrm{~F} / 68$ & Mediastinitis & 71 & 33 & + & - & - & + & - & - & No infection & - & $\mathrm{TN}$ \\
\hline 5 & $\mathrm{M} / 63$ & Septic emboli & 7 & 3 & + & + & - & - & - & Chest/abdomen & Pneumonia & Autopsy & $\mathrm{TP}$ \\
\hline 6 & $\mathrm{M} / 71$ & FUO & 34 & 31 & + & + & - & - & - & - & No infection & - & $\mathrm{TN}$ \\
\hline 7 & $\mathrm{M} / 56$ & FUO & 66 & 30 & + & - & - & + & - & Chest/abd/sinus & No infection & - & $\mathrm{TN}$ \\
\hline 8 & $\mathrm{~F} / 68$ & Septic emboli & 2 & - & - & - & - & + & - & - & Meningitis & Culture & $\mathrm{TN}$ \\
\hline 9 & $\mathrm{M} / 54$ & Septic emboli & 27 & 7 & + & + & - & + & - & - & Candida abscess & Radiology & $\mathrm{TP}$ \\
\hline 10 & $\mathrm{M} / 72$ & Mediastinitis & 35 & 27 & + & - & + & + & - & - & No infection & - & $\mathrm{TN}$ \\
\hline 11 & $\mathrm{M} / 17$ & FUO & 6 & 6 & + & + & + & + & + & - & Dental abscess & Culture & $\mathrm{TP}$ \\
\hline 12 & $\mathrm{~F} / 59$ & FUO & 36 & 4 & + & - & + & - & - & Chest & No infection & - & $\mathrm{TN}$ \\
\hline 13 & $\mathrm{M} / 70$ & FUO & 31 & 17 & + & - & + & - & - & Sinus/chest/abd & Prostatitis & Culture & $\mathrm{TP}$ \\
\hline 14 & F/66 & Septic emboli & 4 & - & + & - & + & - & - & Chest/abdomen & Arthritis & Culture & $\mathrm{TP}$ \\
\hline 15 & $\mathrm{M} / 50$ & $\begin{array}{l}\text { Infected fluid } \\
\text { collection }\end{array}$ & 16 & 16 & + & - & - & - & - & Chest/abdomen & Peritonitis & Culture & $\mathrm{TP}$ \\
\hline 16 & $\mathrm{M} / 58$ & $\begin{array}{l}\text { Infected fluid } \\
\text { collection }\end{array}$ & 14 & - & + & - & - & - & - & Chest/abdomen & No infection & - & $\mathrm{TN}$ \\
\hline 17 & $\mathrm{M} / 42$ & Septic emboli & 10 & 10 & + & - & - & + & - & - & No infection & - & FP \\
\hline 18 & $\mathrm{M} / 68$ & SIRS & 17 & 2 & + & - & - & - & - & - & Pneumonia & Radiology & $\mathrm{TP}$ \\
\hline 19 & $\mathrm{M} / 51$ & FUO & 20 & 28 & + & - & - & - & - & Head/chest & Thyroid abscess & Culture & $\mathrm{TP}$ \\
\hline 20 & $\mathrm{M} / 37$ & FUO & 27 & 26 & + & - & + & - & - & - & No infection & - & FP \\
\hline 21 & $\mathrm{~F} / 68$ & FUO & 17 & 11 & + & - & - & - & - & Head/abdomen & No infection & - & $\mathrm{TN}$ \\
\hline 22 & $\mathrm{M} / 66$ & FUO & 55 & 11 & + & - & - & + & - & - & Sinusitis & Culture & $\mathrm{TP}$ \\
\hline 23 & $\mathrm{M} / 49$ & Septic emboli & 22 & 12 & + & - & - & + & - & - & Osteomyelitis & Culture & $\mathrm{TP}$ \\
\hline 24 & $\mathrm{~F} / 69$ & Septic emboli & 8 & 8 & + & - & - & + & - & - & $\begin{array}{l}\text { Infected hip } \\
\text { prosthesis }\end{array}$ & Culture & $\mathrm{TP}$ \\
\hline 25 & $\mathrm{M} / 70$ & FUO & 48 & 6 & + & - & - & - & - & - & No infection & Biopsy & $\mathrm{TN}$ \\
\hline 26 & $\mathrm{M} / 35$ & FUO & 12 & 27 & + & - & - & - & + & Head/chest & Sinusitis & Clinical picture & $\mathrm{TP}$ \\
\hline $27 \mathrm{a}$ & $\mathrm{M} / 59$ & SIRS & 71 & 8 & + & - & - & + & - & - & Pneumonia & Radiology & $\mathrm{TP}$ \\
\hline $27 \mathrm{~b}$ & $\mathrm{M} / 59$ & SIRS & 148 & - & + & - & + & - & - & - & Leg abscess & Culture & $\mathrm{TP}$ \\
\hline 28 & $\mathrm{~F} / 51$ & Septic emboli & 21 & 20 & + & - & + & - & - & - & Candida abscess & Radiology & $\mathrm{TP}$ \\
\hline 29 & $\mathrm{M} / 1 \mathrm{mo}$ & $\begin{array}{l}\text { Septic } \\
\quad \text { emboli }\end{array}$ & 27 & - & + & - & + & - & - & $\begin{array}{l}\text { Chest/ } \\
\text { abdomen }\end{array}$ & No infection & - & $\mathrm{TN}$ \\
\hline 30 & $\mathrm{M} / 14$ & FUO & 8 & 20 & + & - & + & - & - & - & Lymphoma & Biopsy & $\mathrm{TP}$ \\
\hline 31 & $\mathrm{M} / 1 \mathrm{mo}$ & FUO & 34 & 7 & + & - & + & - & - & - & Infected thrombus & Radiology & $\mathrm{TP}$ \\
\hline 32 & $\mathrm{~F} / 1$ & FUO & 20 & 14 & + & - & + & - & - & Chest/abdomen & No infection & - & $\mathrm{TN}$ \\
\hline 33 & $\mathrm{~F} / 3$ & Septic emboli & 28 & 23 & + & - & + & - & - & - & Candida abscess & Culture & $\mathrm{TP}$ \\
\hline
\end{tabular}

In all patients, blood cultures and chest X-rays were taken. A + sign denotes performed, but are not depicted in the table. $S$ sputum, $B$ broncho-alveolar that the specified test was performed, the - sign that the specified test was not been performed. In all patients blood cultures and a chest X-ray were fluid, $U$ urine, $U S$ ultrasound, $T P$ true positive, $T N$ true negative, $F P$ false positive $F N$ false negative

Table $34 \times 4$ diagram of FDG-PET/CT results compared to follow-up

\begin{tabular}{lccc}
\hline & Follow-up + & Follow-up- & Total \\
\hline FDG-PET/CT+ & 21 & 3 & 24 \\
FDG-PET/CT - & 0 & 11 & 11 \\
Total & 21 & 14 & 35 \\
\hline
\end{tabular}

exclude metastatic abscesses, showed FDG uptake in the pericardium, suggestive of pericarditis. A pericardiocentesis was performed after antibiotic treatment for 18 days, but culture of the fluid remained sterile. During follow-up, this patient became afebrile, without clinical signs of infective pericarditis. A FDG-PET/CT scan of another patient with persistent fever, who had a single positive blood culture with $C$. albicans 7 days prior to the FDGPET/CT scan and who was treated with anti-fungal therapy, showed FDG uptake in the trochanteric bursa. A consulted orthopedic surgeon did not find clinical signs of bursitis, and no further diagnostic tests were performed. Although an infectious bursitis was not formally excluded, the result of this FDG-PET/CT scan was also considered false positive. The third false-positive patient was 

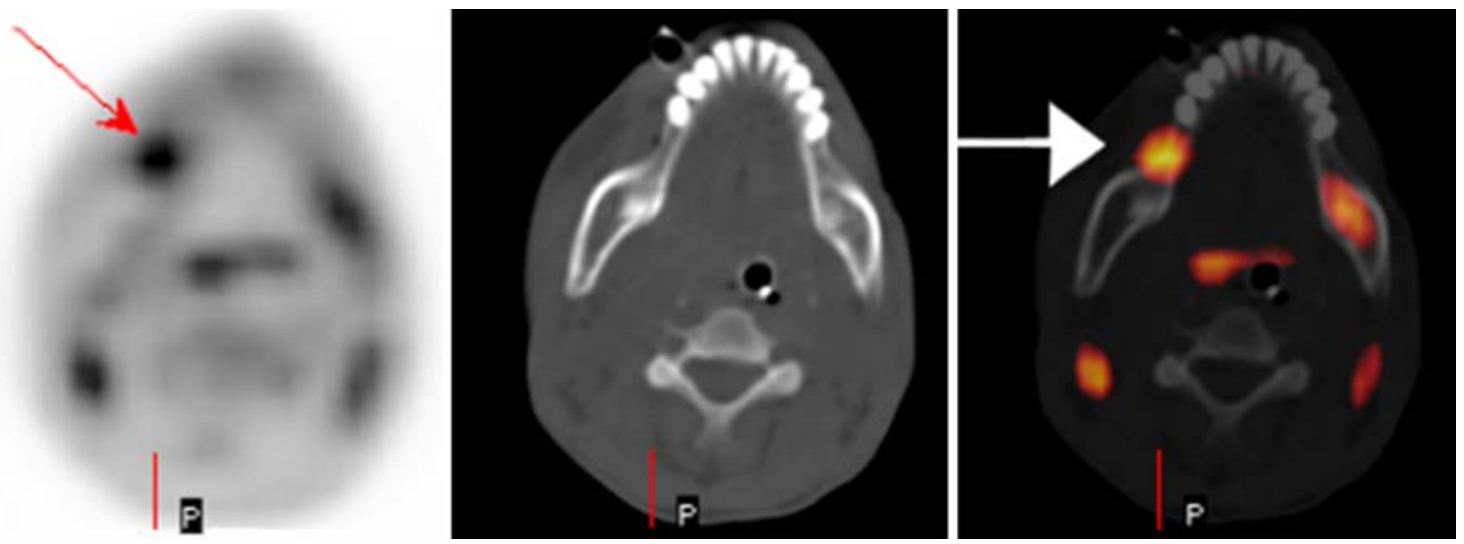

Fig. 1 This 17-year-old male with sickle cell trait was admitted to the ICU because of sepsis. He was treated with piperacilline/ tazobactam, and blood cultures grew Streptococcus milleri. After 9 days, a FDG-PET/CT scan was performed because of persistent fever, which showed increased FDG uptake in the right mandible, suspect for a dental abscess. A dental surgeon subsequently confirmed a dental abscess, which was drained successfully, after which the patient improved rapidly
Fig. 2 This 51-year-old patient had recently received an allogenic stem cell transplantation because of myelodysplastic syndrome. He was admitted to the ICU with respiratory failure. A FDG$\mathrm{PET} / \mathrm{CT}$ scan was performed after 3 weeks of ICU admission because of fever that persisted despite antibiotics and antifungal treatment, showing abnormal accumulation of the tracer in the right thyroid lobe, suggestive of an abscess.

A subsequent hemithyroidectomy confirmed the presence of an abscess. Cultures of this material showed C. albicans

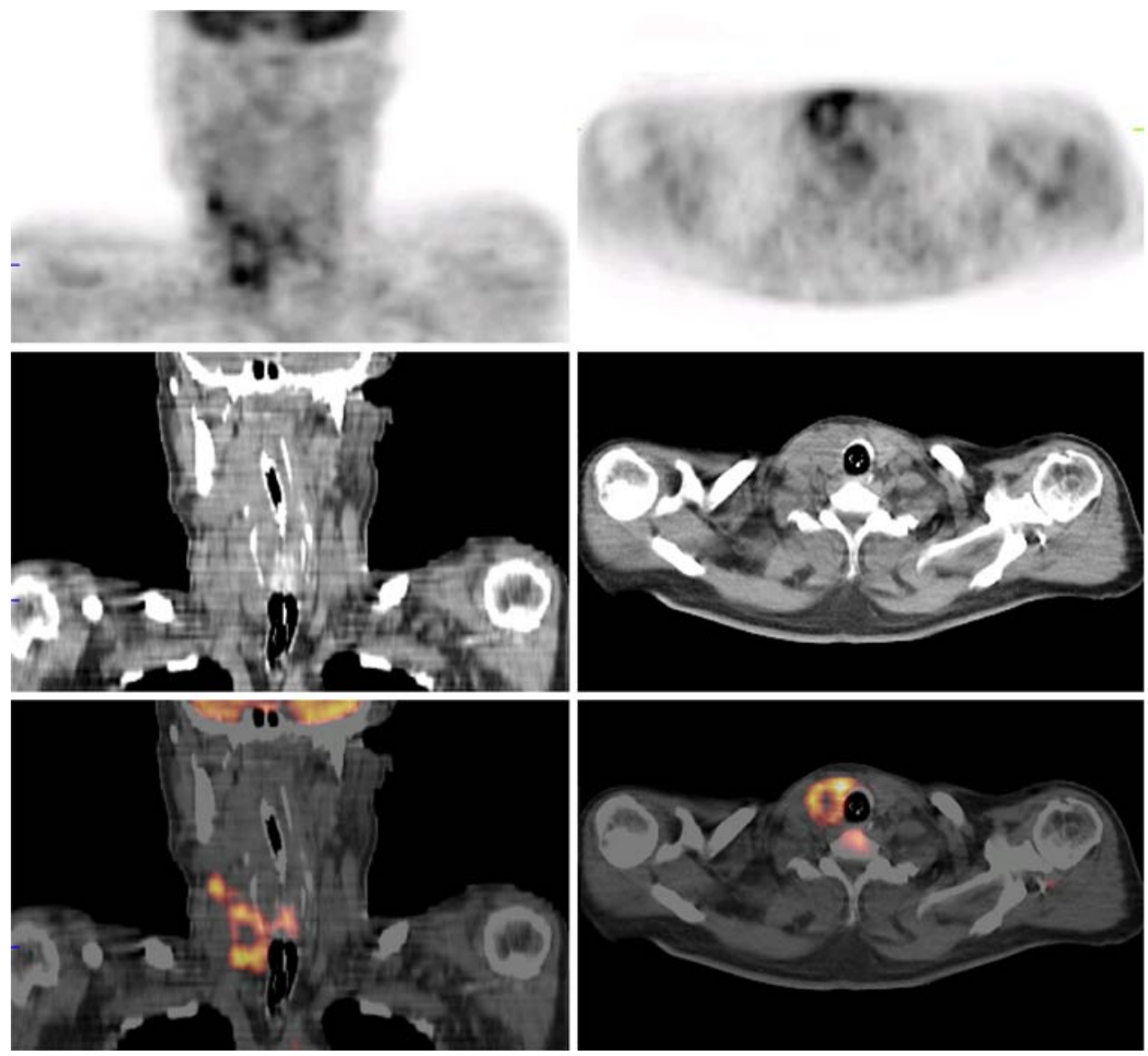

admitted to the ICU with a subarachnoid hemorrhage and had a FDG-PET/CT scan performed after 4 weeks of spiking fever with repeated positive blood cultures with coagulase-negative staphylococci, for which he was treated with teicoplanin for more than 2 weeks. The FDG-
PET/CT scan showed abnormal FDG uptake consistent with an infected thrombus in the internal jugular vein with signs of inflamed lymph nodes in the surrounding tissues. Teicoplanin was stopped 3 days hereafter, and the patient was discharged from the ICU 2 days later. In the 4 weeks 
Table 4 Diagnostic methods establishing the final infectious diagnosis

\begin{tabular}{lll}
\hline Diagnostic method & \multicolumn{2}{l}{ No. of cases } \\
\cline { 2 - 3 } & $\begin{array}{l}\text { FDG-PET/CT } \\
\text { abnormal }\end{array}$ & $\begin{array}{l}\text { FDG-PET/CT } \\
\text { scan normal }\end{array}$ \\
\hline Definite & 12 & 1 \\
Culture or serology & 2 & 1 \\
Biopsy & 1 & - \\
Autopsy & & - \\
Probable & 5 & \\
Radiology & 1 & \\
Clinical picture & 1 & \\
\hline
\end{tabular}

of follow-up at the ward, fever gradually disappeared, and the PET results were categorized as false positive. Based on these results, sensitivity of FDG-PET was $100 \%$, specificity was $79 \%$, positive predictive value was $88 \%$ and negative predictive value was $100 \%$. Overall accuracy of the FDG-PET scans was $91 \%$. The positive likelihood ratio was 4.8 , and the negative likelihood ratio was 0 . Solely based on biopsies and cultures, similar results were obtained: sensitivity of $100 \%$, a specificity of $78 \%$, a PPV of $84 \%$ and a NPV of $100 \%$.

\section{Discussion}

In this clinical study we demonstrated the feasibility and additional value of combined FDG-PET/CT scans in ventilated critically ill patients. The high sensitivity and specificity are promising, considering the fact that the patients represent a subgroup of critically ill patients in which the absence or presence of an infection could not be obtained with conventional diagnostic procedures. Naturally, identification of the site of infection is essential to institute the most appropriate therapy. The high specificity of $79 \%$ indicates that in this small, highly selected group of patients in whom no diagnosis could be reached with conventional diagnostics, FDG-PET/CT scanning appears to be of additional value in detecting focal infection and inflammation. Although one may argue that, e.g., sinusitis or pneumonia could be diagnosed by CT alone, CT scans of the sinuses frequently show air-fluid levels whose clinical relevance is unclear, and chest CT scans frequently show pulmonary abnormalities consistent with atelectasis, pleural effusion and infiltrates in ventilated ICU patients. In our opinion, the addition of PET scanning to detect increased metabolism consistent with inflammation in these lesions has additional value in deciding whether or not the found abnormalities on the CT scan are actually regions of infection and the cause of symptoms and signs of the patient.
The high negative predictive value is also of major clinical importance to exclude a clinically important infection and prevent patients from being treated with antibiotics or undergoing unnecessary invasive procedures. As a measure of the post-test probability compared to the estimated pre-test probability of an infection the positive and negative likelihood ratio was calculated, showing that the FDG-PET/CT scan is of evident additional clinical value to indicate and exclude patients with the disease.

Up to now, scintigraphic imaging has played a very limited role in detecting suspected focal infections in ICU patients, as only two ICU case series are available in the literature. In a retrospective study, Kao et al. [20] investigated the value of Tc-99m labeled leucocytescintigraphy in detecting a source for occult sepsis in 25 ICU patients, demonstrating a sensitivity of $96 \%$ and a specificity of $84 \%$. However, sensitivity and specificity were not calculated per patient, but per body region, resulting in more true negative sites and thus a higher specificity. Minoja et al. [21] prospectively evaluated the use of Tc-99m-labeled leukocyte scintigraphy in detecting the cause of occult sepsis in a group of 26 surgical and trauma patients and found a sensitivity and specificity of 95 and $91 \%$, respectively. It is important to note that scintigraphic scans using Ga-67, In-111-labeled or Tc-99m labeled leukocytes have disadvantages, such as the amount of blood needed to harvest enough leukocytes (up to $120 \mathrm{ml}$ ), handling of potentially infected blood products, high radiation burden, and the long time span between injection and diagnosis. Advantages of FDGPET are higher resolution, sensitivity in chronic lowgrade infections and high accuracy in the central skeleton [22]. Furthermore, the time between FDG-injection and diagnosis is approximately $1 \mathrm{~h}$, so results are available much sooner than in case of other nuclear medicine techniques.

Our study is limited by several factors. First, in some cases there was a specific suspicion of a localized infection, possibly increasing the a priori chance of an infection. However, we chose to describe the daily standard practice in this observational study, thereby accepting the differences in indications of PET/CT scanning. Second, a final diagnosis could not be established in all patients. This is especially important in the group of patients with negative FDG-PET/CT scans, since missing a diagnosis of focal infection can have serious consequences. Nevertheless, in none of the 11 patients with a negative FDG-PET/CT scan was a subsequent infectious diagnosis reached. When additional diagnostic procedures showed normal results and follow-up did not reveal a diagnosis, in the absence of a real gold standard, it seemed reasonable to presume that a focal infection with therapeutic consequences was not the cause of the signs or symptoms of the patient. However, several patients with normal FDG-PET/CT scans did receive 
short courses of antibiotic treatment, so the presence of an infection cannot be ruled out completely. In addition, some of the patients with a normal FDG-PET/CT scan developed an infectious complication more than 2 weeks later during their ICU stay. It is difficult to exactly determine whether these infectious processes could have been present at the time of the FDG-PET/CT scan. However, because of the fact that all other diagnostic tests performed at the time of the FDG-PET/CT scans in these patients were also normal, we considered it unlikely that these infections were present at the time of the FDG-PET/ CT scan, and we considered these FDG-PET/CT scans true negative when no focal infection was diagnosed in the 2 weeks following the scan. Despite these limitations, our results indicate that a normal FDG-PET/CT scan excludes an important infectious process needing drainage or prolonged antibiotic treatment. To our knowledge, this is the first study evaluating the additional value of FDG-PET/CT scanning in diagnosing focal infections in ventilated critically ill patients. In accordance, studies evaluating PET scanning in diagnosing FUO in noncritically ill patients showed comparable results [14-16, 23-25]. Our group previously and more specifically investigated the role of FDG-PET in a subgroup of 48 non-critically ill, hospitalized patients with a suspected focal infection or inflammatory disease and found that 38 out of 55 FDG-PET scans were abnormal, of which 36 correctly pointed to the cause of the symptoms. In addition, a negative predictive value of $100 \%$ was found [16] These findings indicate that in both non-ICU as well as critically ill ICU patients a negative PET/CT scan appears to exclude a focal infection almost certainly as a cause of the clinical symptoms.

In conclusion, our experience demonstrates the feasibility and potential value of the FDG-PET/CT scan, and it appears to be a promising tool in detecting and excluding focal infection in critically ill patients. Since the exact role of FDG-PET/CT scanning in critically ill ICU patients is yet undefined, we have to be vigilant to potential hazardous additional diagnostic consequences of the results of this technique. Nevertheless, the patient that underwent a pericardiocenthesis that turned out to be negative was the only case of the study where an additional invasive procedure was performed with a clear detrimental risk/benefit ratio. In this specific case the decision to perform a pericardiocenthesis was not only based on the results of the FDG-PET/CT scan, but also on the results of echocardiography. In the other 13 patients with a positive FDG-PET/CT scan, subsequent diagnostic tests resulted in the final diagnosis.

We propose that the use of the FDG-PET/CT scan should be considered earlier in critically ill patients suspected of an infection. Whether FDG-PET/CT scanning can be of additional value in the early detection of specific types of focal infection notoriously difficult to diagnose (e.g., mediastinitis, metastatic abscesses) will be the subject of future research.

Open Access This article is distributed under the terms of the Creative Commons Attribution Noncommercial License which permits any noncommercial use, distribution, and reproduction in any medium, provided the original author(s) and source are credited.

\section{References}

1. Guhlmann A, Brecht-Krauss D, Suger G, Glatting G, Kotzerke J, Kinzl L, Reske SN (1998) Fluorine-18-FDG PET and technetium-99 m antigranulocyte antibody scintigraphy in chronic osteomyelitis. J Nucl Med 12:2145-2152

2. Guhlmann A, Brecht-Krauss D, Suger G, Glatting G, Kotzerke J, Kinzl L, Reske SN (1998) Chronic osteomyelitis: detection with FDG PET and correlation with histopathologic findings. Radiology 3:749-754

3. Kalicke T, Schmitz A, Risse JH, Arens S, Keller E, Hansis M, Schmitt O, Biersack HJ, Grunwald F (2000) Fluorine-18 fluorodeoxyglucose PET in infectious bone diseases: results of histologically confirmed cases. Eur J Nucl Med 5:524-528
4. Bleeker-Rovers CP, Bredie SJ, van der Meer JW, Corstens FH, Oyen WJ (2003) F-18-fluorodeoxyglucose positron emission tomography in diagnosis and follow-up of patients with different types of vasculitis. Neth J Med 10:323-329

5. Bleeker-Rovers CP, Bredie SJ, van der Meer JW, Corstens FH, Oyen WJ (2004) Fluorine 18 fluorodeoxyglucose positron emission tomography in the diagnosis and follow-up of three patients with vasculitis. Am J Med 1:50-53

6. Belhocine T, Blockmans D, Hustinx R, Vandevivere J, Mortelmans L (2003) Imaging of large vessel vasculitis with (18)FDG PET: illusion or reality? A critical review of the literature data. Eur J Nucl Med Mol Imaging 9:1305-1313
7. Turlakow A, Yeung HW, Pui J, Macapinlac H, Liebovitz E, Rusch V, Goy A, Larson SM (2001) Fludeoxyglucose positron emission tomography in the diagnosis of giant cell arteritis. Arch Intern Med 7:1003-1007

8. De Winter F, Petrovic M, Van de WC, Vogelaers D, Afschrift M, Dierckx RA (2000) Imaging of giant cell arteritis: evidence of splenic involvement using FDG positron emission tomography. Clin Nucl Med 8:633-634

9. Bicik I, Bauerfeind P, Breitbach T, von Schulthess GK, Fried M (1997) Inflammatory bowel disease activity measured by positron-emission tomography. Lancet 9073:262

10. Skehan SJ, Issenman R, Mernagh J, Nahmias C, Jacobson K (1999) 18Ffluorodeoxyglucose positron tomography in diagnosis of paediatric inflammatory bowel disease. Lancet 9181:836-837 
11. Neurath MF, Vehling D, Schunk K, Holtmann M, Brockmann H, Helisch A, Orth T, Schreckenberger M, Galle PR, Bartenstein P (2002) Noninvasive assessment of Crohn's disease activity: a comparison of $18 \mathrm{~F}$ -

fluorodeoxyglucose positron emission tomography, hydromagnetic resonance imaging, and granulocyte scintigraphy with labeled antibodies. Am J Gastroenterol 8:1978-1985

12. Brudin LH, Valind SO, Rhodes CG, Pantin CF, Sweatman M, Jones T, Hughes JM (1994) Fluorine-18 deoxyglucose uptake in sarcoidosis measured with positron emission tomography. Eur J Nucl Med 4:297305

13. Lewis PJ, Salama A (1994) Uptake of fluorine-18-fluorodeoxyglucose in sarcoidosis. J Nucl Med 10:1647-1649

14. Meller J, Altenvoerde G, Munzel U, Jauho A, Behe M, Gratz S, Luig H, Becker W (2000) Fever of unknown origin: prospective comparison of [18F]FDG imaging with a double-head coincidence camera and gallium-67 citrate SPET. Eur J Nucl Med 11:1617-1625

15. Blockmans D, Knockaert D, Maes A, De CJ, Stroobants S, Bobbaers H, Mortelmans L (2001) Clinical value of [(18)F]fluoro-deoxyglucose positron emission tomography for patients with fever of unknown origin. Clin Infect Dis 2:191-196
16. Bleeker-Rovers CP, de Kleijn EM, Corstens FH, van der Meer JW, Oyen WJ (2004) Clinical value of FDG PET in patients with fever of unknown origin and patients suspected of focal infection or inflammation. Eur J Nucl Med Mol Imaging 1:29-37

17. Jaruskova M, Belohlavek O (2006) Role of FDG-PET and PET/CT in the diagnosis of prolonged febrile states. Eur J Nucl Med Mol Imaging 8:913918

18. Bleeker-Rovers CP, Vos FJ, Wanten GJ, van der Meer JW, Corstens FH, Kullberg BJ, Oyen WJ (2005) 18F-FDG PET in detecting metastatic infectious disease. J Nucl Med 12:2014-2019

19. Boellaard R, Oyen WJ, Hoekstra CJ, Hoekstra OS, Visser EP, Willemsen AT, Arends B, Verzijlbergen FJ, Zijlstra J, Paans AM, Comans EF, Pruim J (2008) The Netherlands protocol for standardisation and quantification of FDG whole body PET studies in multi-centre trials. Eur J Nucl Med Mol Imaging 12:2320-2333

20. Kao CH, Wang YL, Liao SQ, Wang SJ (1992) Tc-99 m HMPAO labelled WBCs in the detection of occult sepsis in the intensive care unit. Intensive Care Med 1:15-19
21. Minoja G, Chiaranda M, Fachinetti A, Raso M, Dominioni L, Torre D, De Palma D (1996) The clinical use of $99 \mathrm{~m}$-Tc-labeled WBC scintigraphy in critically ill surgical and trauma patients with occult sepsis. Intensive Care Med 9:867-871

22. Bleeker-Rovers CP, Boerman OC, Rennen HJ, Corstens FH, Oyen WJ (2004) Radiolabeled compounds in diagnosis of infectious and inflammatory disease. Curr Pharm Des 24:2935-2950

23. Buysschaert I, Vanderschueren S, Blockmans D, Mortelmans L, Knockaert D (2004) Contribution of (18)fluoro-deoxyglucose positron emission tomography to the work-up of patients with fever of unknown origin. Eur J Intern Med 3:151-156

24. Kjaer A, Lebech AM, Eigtved A, Hojgaard L (2004) Fever of unknown origin: prospective comparison of diagnostic value of 18F-FDG PET and 111In-granulocyte scintigraphy. Eur J Nucl Med Mol Imaging 5:622-626

25. Lorenzen J, Buchert R, Bohuslavizki KH (2001) Value of FDG PET in patients with fever of unknown origin. Nucl Med Commun 7:779-783 